\title{
Enhancement of Sander illusion in minimal form
}

RICHARD P. RUNYON ${ }^{1}$ AND MICHAEL R. COOPER C. W. POST COLLEGE

This paper reports the effects of systematically reducing the number of component lines in the Sander parallelograms upon judgments of the relative lengths of the diagonal lines. The illusion was maximal when the parallelogram was reduced to its minimal form, viz., three parallel lines remained with all other lines, including the diagonals, omitted.

There has been a recent resurgence of interest in geometrical illusions, as witnessed by major articles in Psychological Bulletin (Over, 1968) and Scientific American (Gregory, 1968). This renewed interest has been accompanied by a proliferation of theories seeking explanations in terms of physiological processes (Over, 1968). Over concludes, “. . . It cannot be stated with any certainty whether the distortions obtained with illusion figures are dependent on neural inhibitory interaction, information sampling operations, or learning processes [p. 559]."

One possible way to reduce the confusion would be to demonstrate that one or more illusions are special cases of a more fundamental illusion. A satisfactory explanation for the latter would presumably also account for the special cases. With these considerations in mind, the authors conducted an investigation to determine if the Sander parallelogram illusion (see Fig. 1A) might be a special case of the Müller-Lyer illusion. Examination of the Sander illusion reveals that the smaller parallelogram to the right is a special case of the Müller-Lyer illusion with arrowheads set at an acute angle. In the larger parallelogram to the left, the angle of the arrowheads is less acute. Consequently, one should not be surprised that there is greater distortion in judging the length of the right diagonal as opposed to the left. Going one step further, we reasoned that systematically dropping lines so as to reduce the similarity of the Sander illusion to the Muiller-Lyer illusion should lead to a decreased distortion in judging the length of the right diagonal. In the experiment that follows, the horizontal lines in the parallelogram were dropped in Condition B. In Condition C, the illusion was reduced to its minimal form, viz., three parallel lines remained with all other lines, including the diagonals, dropped.
Reasoning from the Muiller-Lyer analogy, this condition (C) would be expected to produce least distortion in judging the length of the diagonals.

\section{EXPERIMENT 1}

Methods

Subjects. Forty-eight undergraduate students (31 male, 17 female) were selected on a voluntary basis from introductory psychology courses.

Apparatus. Ten examples of the Sander illusion for each of the three conditions in which the length of the diagonal B-C varied between $1 / 16$ in. shorter than the diagonal A-B to $24 / 16$ in. greater. All ten illusions were displayed simultaneously on a flat surface in a nonsystematic arrangement.

Design. All Ss received three conditions. The order of presentation was balanced so that all six different presentations occurred an equal number of times. The Ss stood at a distance of approximately $1 \mathrm{ft}$ from the display materials and viewed the stimuli binocularly.

Procedures. In order to obtain a more stable estimate of each S's error score, each set of three illusions was presented twice. The S's score for a given condition was the mean of both presentations. The Ss were instructed to identify the figure in which the physically present or imaginary diagonal $A-B$ appeared to equal $B-C$ in length. No time restrictions were imposed upon the Ss. Each session lasted approximately $10 \mathrm{~min}$.

Results. The mean errors obtained under the three experimental conditions were: Condition A, .612 in.; B, .438 in.; and C, $.788 \mathrm{in}$. These correspond to the following percentages of error: A, $25.2 \%$;, $17.3 \%$; and $\mathrm{C}, 33.0 \%$.

An analysis of variance of the data obtained in Experiment 1 is presented in Table 1. There was a significant effect of experimental treatments and orders, as well as a significant interaction of Conditions by Practice effects. All F-ratios were significant at well beyond the 0.01 level.

Employing Duncan's multiple range test, all differences between pairs of treatment means were found to be significant beyond the 0.01 level. The greatest error is found under the $C$ condition in which the diagonals must be imagined. The standard Sander illusion (Condition A) produced
Table 1

\begin{tabular}{|c|c|c|c|c|}
\hline Source & df & SS & MS & $\mathbf{F}$ \\
\hline $\begin{array}{l}\text { Subjects } \\
\text { : Orders } \\
\text { : Error a }\end{array}$ & $\begin{array}{r}47 \\
5 \\
42\end{array}$ & $\begin{array}{r}13090 \\
7088 \\
6002\end{array}$ & $\begin{array}{r}1417.60 \\
142.90\end{array}$ & $9.92 *$ \\
\hline $\begin{array}{l}\text { Conditions } \\
\text { Practice } \\
\text { Effects }\end{array}$ & 2 & $\begin{array}{r}3015 \\
10\end{array}$ & $\begin{array}{r}1501.50 \\
5.00\end{array}$ & (96.44** \\
\hline $\begin{array}{l}\text { Cond. X PE } \\
\text { Error b }\end{array}$ & $\begin{array}{r}84 \\
88\end{array}$ & $\begin{array}{l}1819 \\
1370 \\
\end{array}$ & $\begin{array}{r}454.75 \\
15.57\end{array}$ & $29.25 * *$ \\
\hline
\end{tabular}

$* * p<0.01$

errors less than Condition $\mathrm{C}$ and greater than Condition B.

The interaction between conditions and practice effects is presented in Fig. 2.

It is clear from examination of Fig. 2 that the differences among the three conditions were small and nonsignificant on the first practice trial. However, the differences clearly manifested themselves in Trials 2 and 3.

\section{Discussion}

Contrary to the original hypothesis, the results indicate that the $C$ condition, in which the Sander illusion was reduced to its minimal form, produced the greatest
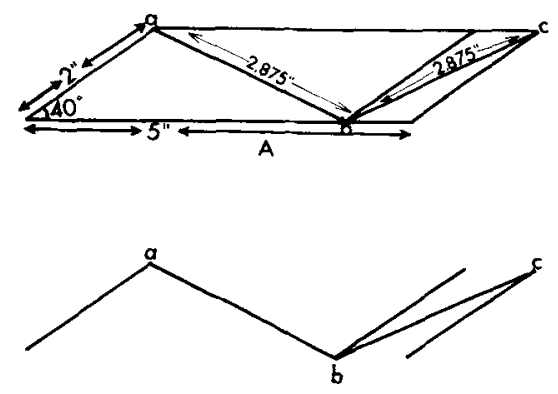

B
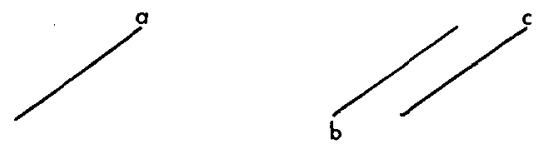

C

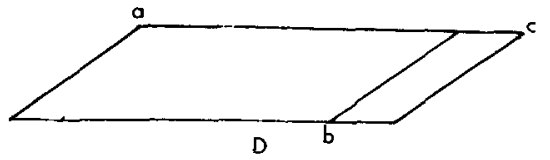

Fig. 1. The standard stimulus presented in each condition. 

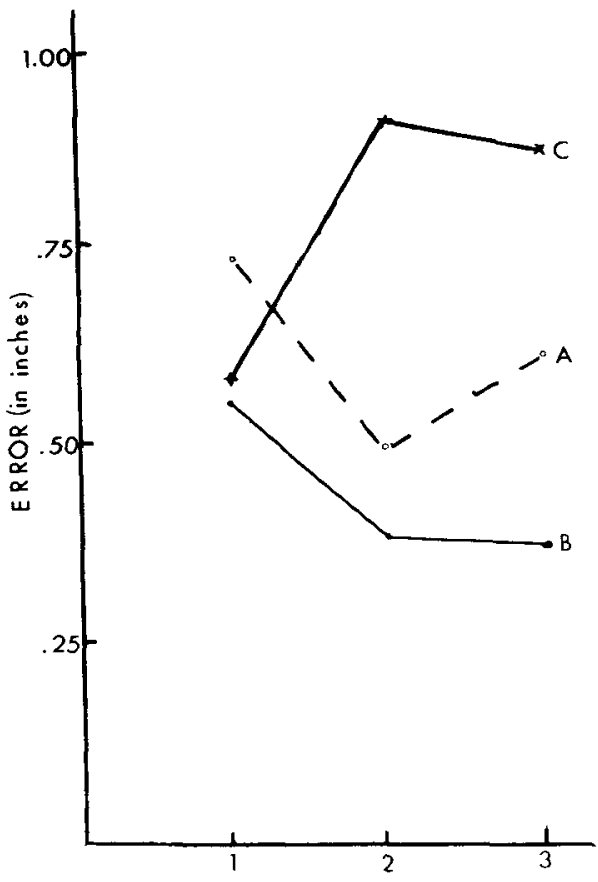

ORDINAL POSITION

Fig. 2. Mean error as a function of the interaction between conditions and practice effects.

distortion in estimating the length of the B-C diagonal relative to the length of the A-B diagonal. Indeed, the magnitude of the error was fully $29 \%$ greater than in the standard form of the Sander illusion.

This result is surprising. Gregory (1966) reports that a similar impression of the Müller-Lyer illusion, in which the shaft position between fins is removed, leads to a diminished error. We have since conducted a study, employing the Muiller-Lyer illusion that challenges Gregory's observation (Cooper \& Runyon, 1969) ${ }^{2}$.

In interpreting the results of Experiment 1, it is not clear whether the increased distortion in judging the length of the diagonal B-C stems from the elimination of the diagonals, forcing the $S s$ to imagine these diagonals, or from the elimination of the horizontal lines since both of these characteristics pertain to Condition C. It is possible that the increased illusion stems from either of these sources. Experiment 2 was undertaken to compare the elimination of the diagonals in the standard Sander illusion to the elimination of both the diagonals and the enclosing lines (The $\mathrm{C}$ condition in Experiment 1).

\section{EXPERIMENT 2}

\section{Method}

Subjects. Thirty-six undergraduate students drawn from introductory psychology classes (20 males; 16 females) were Ss.

Apparatus. Ten illusions for each of the conditions (C and $D$ ) in which the length of the diagonal $B-C$ varied between $1 / 16$ in. shorter than the diagonal A-C to $24 / 16$ in. longer. All ten illusions were displayed simultaneously on a flat surface in a nonsystematic arrangement.

Design. Both orders of presentation of the two treatments were employed, $18 \mathrm{Ss}$ receiving Order $\mathrm{CD}$ and 18 receiving Order DC.

Procedure. Same as in Experiment 1 except that only two sets of illusions were employed.

Results. An analysis of variance for the data obtained in Experiment 2 is presented in Table 2.

The $\mathrm{C}$ condition produced significantly greater errors than the $D$ condition, $\mathrm{P}<0.01$. Th respective mean errors were: Condition C, 1.26 in.; Condition D, $1.17 \mathrm{in}$. These correspond to the following percentages of error: $\mathrm{C}=58.0 \% ; \mathrm{D}=52.6 \%$.

\section{DISCUSSION}

The second experiment demonstrated that the illusion is maximal when Ss had to imagine both the diagonals and the horizontal lines of the parallelogram.

The results of these experiments raise serious questions concerning explanations of illusions that have been offered in recent literature.

Several physiological theories noted by Over claim that perceptual distortion "occurs whenever the nervous system signals information about spatially adjacent contours [1968, p. 545]." According to this theory, increased inputs should lead to greater input complexity and greater error scores. The reverse held to be true in the above experiments, i.e., the error scores were maximum when the illusion figure was presented in a form in which all spatially adjacent contours were eliminated.

Motokawa (1950) offered a retinal induction explanation of illusions, reporting induction fields for several geometrical illusions. Filled space was found to produce a more extensive illusion than unfilled space. Motokawa considers this fact to be the basis of perceptual overestimation of filled space. In our studies, the minimal form of the Sander illusion may be considered to con tain more unfilled space. Yet the illusion was maximum under these conditions.

One might hypothesize that the removal of the diagonals in the $\mathrm{C}$ condition eliminates the possibility of a direct comparison of existing lines. The greater error in the minimal form of the Sander illusion may therefore represent faulty
Table 2

Analysis of Variance: Experiment 2

\begin{tabular}{lrrrr}
\hline Source & df & \multicolumn{1}{c}{ SS } & \multicolumn{1}{c}{ MS } & \multicolumn{1}{c}{ F } \\
\hline Subjects & 35 & 8695 & 248.43 & \\
Conditions & 1 & 93 & 93.00 & $13.74 * *$ \\
Error & 35 & 237 & 6.77 & \\
Total & 71 & 9025 & & \\
$* * \quad p<0.01$ & & &
\end{tabular}

judgment due to the lack of an objective referent. A study recently completed by the authors (Cooper \& Runyon, 1969²) employing the Müller-Lyer illusion, questions this interpretation. We found an interaction in which the fins-out condition decreases the error score when the shaft is removed but the fins-in condition increases the error.

One unexpected difference that we cannot account for was the fact that the illusion in Experiment 2 produced a far greater error score than in Experiment 1. Indeed, the mean error approached the maximal value possible, suggesting that cited error might have been greater where there a wider range of differences at the end of the scales. We have no way of accounting for the magnitude of the differences in Experiments 1 and 2 except to suggest that, by chance, different populations were sampled. In Experiment 1, for example, there were six known mathematics majors all of whom demonstrated virtually no error score under any of the conditions.

It is possible that the standard form of the Sander illusion, which was employed only in Experiment 1, exerts some sort of "anchoring" effect, causing Ss to modify downward their estimates of the remaining illusions. Additionally, it is possible that $t$ he standard form of the illusion encourages a "whole viewing" attitude which enhances the illusion obtained with the Muiller-Lyer figure (Bates, 1923).

\section{REFERENCES}

BATES, M. A study of the Müller-Lyer illusion, with special reference to paradoxical movement and the effect of attitude. American Journal of Psychology, 1923, 34, 46-72.

GREGORY, R. L. Eye and brain. Linden: Weidenfeld \& Nicholson, 1966.

GREGORY, R. L. Visual illusions. Scientific American, 1968, 219, 66-76.

MOTOKAWA, K. Field of retinal induction and optical illusion. Journal of Neurophysiology, $1950,20,408-434$.

OVER. Explanations of geometrical illusions. Psychological Bulletin, 1968, 70, 545-562.

\section{NOTES}

1. Address: Psychology Department, C. W. Post College, Greenvale, New York 11548.

2. Cooper, M., \& Runyon, R. Error increase and decrease in minimal form of Muller-Lyer illusion. 1969, in preparation.

(A ccepted for publication, November 15, 1969.) 